\title{
Zwischen Green Growth und De-Growth
}

\author{
Der Zusammenhang von Wachstum und \\ Nachhaltiger Entwicklung steht im Kern einer \\ Nachhaltigkeitsökonomik. Um eine Rückführung \\ umweltbelastender Mengenströme zu erreichen, \\ müssen zunächst die Wachstumstreiber für Kon- \\ sumenten und Produzenten identifiziert werden. \\ Von Frank Beckenbach, Bernd Wagner \\ und Heinz Welsch
}

\footnotetext{
pätestens seit den 1970er Jahren wird das Verhältnis von Wachstum und Nachhaltigkeit systematisch und, insbesondere auch durch die Ressourcenökonomik, konzeptionell diskutiert. Dabei stand die Verfügbarkeit von Naturgütern als Grenze des Wachstums im Fokus. Inzwischen hat sich in Teilen der Wirtschaftswissenschaften diese Perspektive umgekehrt. Inwieweit ist Wachstum eine Bedrohung für Nachhaltigkeit im Sinne des Erhalts natürlicher Ressourcen?

Im Kern geht es bei dieser Frage vor allem um den intergenerativen Interessenausgleich bei der Nutzung natürlicher Ressourcen. Wie viele und welche Assets soll die heutige Generation den zukünftigen Generationen durch Investition bereitstellen beziehungsweise durch Nutzungsverzicht hinterlassen? Die Beantwortung dieser Frage hängt davon ab, inwieweit Naturkapital durch Sachkapital und Humankapital substituiert werden kann. Während beispielsweise fossile Energieträger durch Investitionen in regenerative Erzeugungstechnologien substituiert werden können, ist eine Substitution ganzer Ökosysteme, wie des Klimasystems, der Biodiversität wie auch des Ackerbodens nach dem gegenwärtigen Wissensstand nicht erreichbar und abgesehen davon nach allgemeinem Konsens auch nicht erstrebenswert.

Aus diesen Gründen wird das Spannungsverhältnis zwischen Wachstum und Nachhaltigkeit am stärksten deutlich bei der Betrachtung von Ökosystemen. Dieses Spannungsverhältnis lässt sich aus der Sicht der Nachhaltigkeitsökonomik dergestalt spezifizieren, dass die mit wirtschaftlichen Aktivitäten verbundenen Nutzungen beziehunsgweise Belastungen von Quellen und Senken im Blick auf die damit verbundenen Gefährdungen ökosystemischer Leistungen abgeschätzt werden. Dies impliziert, dass das mit den wirtschaftlichen Aktivitäten verbundene Anwachsen und Ausdifferenzieren der Mengenströme, zum Beipsiel von Rohstoffen, Emissionen oder Abfall, den Kern des Problems ausmacht.
}

Bei der Identifikation der Treiber für diese Mengendynamik steht die Nachhaltigkeitsökonomik noch am Anfang. Ein erster Zugang zu diesem Forschungsbereich lässt sich mit Hilfe der sogenannten IPAT-Identität eröffnen. Danach setzt sich die zunehmende Belastung natürlicher Systeme aus den Faktoren Bevölkerungswachstum, Wachstum der Pro-Kopf- Produktion und der Umwelt- und Ressourcenintensität der Produktion zusammen, also im Kern aus den Komponenten „Scale“ und „Efficiency“. Allerdings sind mit dieser aggregierten Identitätsbetrachtung weder die heterogenen Mengenströme erfasst, die der Umweltbelastung zugrunde liegen, noch die kausalen Abhängigkeiten zwischen Produktion und Bevölkerung beziehungsweise zwischen Umweltbelastung und Produktion. Dies erfordert nicht nur ein Hinausgehen über diese aggregierte Betrachtungsweise, sondern auch die Einbeziehung spezifisch historischer Bedingungen der Produktions- und Konsumweise.

Erst aus einer derart vertieften Betrachtung lassen sich Anhaltspunkte für die Beantwortung der Frage gewinnen, ob beziehungsweise inwieweit eine Entkoppelung der Naturbelastung von Produktion und Konsum möglich ist. Erstens stellt sich die Frage, ob es möglich ist, die umweltbezogen Mengenströme und die damit verbundene Belastung auf einem Niveau zu halten, das die Leistungsfähigkeit der wichtigen Ökosysteme weiterhin sicherstellt? Zweitens, ob mit dieser Anforderung ein Wachstum der ökonomischen Aktivitäten (Green Growth) kompatibel ist oder aber dafür eine Drosselung oder gar Rückführung des Wachstums dieser Aktivitäten notwendig ist, um Überleben auf Dauer zu gewährleisten (De-Growth)?

Während für einige einzelne Schadstoffe Entkoppelungstendenzen im Sinne des Green Growth empirisch nachweisbar sind, stellt sich die Frage, ob solche partiellen Entlastungen nicht lediglich Verlagerungen hin zu anderen Formen der Umweltbelastung darstellen. Ferner ist eine solche Entlastung für das Klimasystem und die Biodiversität sowie die Verknappung und Übernutzung des Bodens derzeit nicht erkennbar.

\section{Forschungsagenda zu den Wachstumstreibern}

Die für die Beantwortung der genannten Fragen erforderliche Identifikation der Treiber für die Dynamik der mit den wirtschaftlichen Aktivitäten verbundenen Mengenströme kann auf der Ebene des Individuums, also des Konsumenten, der Unternehmung beziehungsweise des Produzenten und des Staates sowie deren Zusammenwirken ansetzen.

Auf der Ebene des Individuums lassen sich zum einen Bestrebungen zur Ausdehnung des Konsums identifizieren, die $\rightarrow$ 
mit der Produktions- und Lebensweise in modernen Marktwirtschaften zusammenhängen:

- ungesättigte Bedürfnisse insbesondere bei Beziehern von geringem Einkommen (nachholender Konsum),

- das Bedürfnis, dem Standard der sozialen Bezugsgruppe zu entsprechen (konformistischer Konsum) oder diesen sogar zu übertreffen (positioneller Konsum) und

- das Bedürfnis, Verschlechterungen der Lebensumgebung zu kompensieren (defensiver Konsum).

Abgesehen von der genaueren Betrachtung dieser Konsumtreiber oder -zwänge geht es um die Erforschung der Rolle von Präferenzen für Konsumzuwachs im Gegensatz zu Präferenzen für Naturbewahrung. Hier haben die Verhaltensökonomik und die ökonomische Zufriedenheitsforschung gezeigt, dass zumindest in Industrieländern Präferenzen sich weniger auf absolute Payoffs beziehungsweise Konsumlevels richten, sondern auf Payoffs relativ zu Referenzwerten, die insbesondere das in der Vergangenheit erreichte Niveau reflektieren. Wachstum von Einkommen und Konsum schaffen zwar nicht mehr Glück oder Zufriedenheit, scheinen aber notwendig, um die Zufriedenheit konstant zu halten. Wichtige und weitgehend offene Fragen sind, ob diese in westlich geprägten Ländern zu findende Wert- und Präferenzstruktur gewachsen ist, ob sie reversibel ist und inwieweit ein internationaler und interkultureller Transfer dieser Werte in andere Gesellschaften stattfindet. Es stellt sich die Frage, ob nicht bereits dieser, kaum zu steuernde, aber absehbare Transfer, Wachstumstreiber beinhaltet, die ökologische Belastungskapazitäten überschreiten.

Auf der Ebene der Unternehmung lassen sich die Treiber für die Dynamik der Mengenströme zum einen direkt aus dem Marktwettbewerb ableiten:

- Ein probates Mittel für die Senkung der Stückkosten ist die Erhöhung der Produktionsmenge (steigende Skalenerträge);

- Unternehmen die Innovation betreiben, sind gezwungen, durch die extensive Anwendung der Innovationsergebnisse Wettbewerbsvorteile zu erlangen beziehungsweise die Innovationskosten $\mathrm{zu}$ amortisieren;

- schließlich werden Unternehmen unter Wettbwerbsbedingungen in der Regel die Strategie einer Vergrößerung des Marktanteils verfolgen.

Zum andern sind die unternehmensbezogenen Treiber für die Mengenströme aus der geld- und finanzmarktlichen Einbettung der Unternehmensaktivitäten zu erklären. Hier ist nicht nur, im Falle der Fremdfinanzierung, die Refinanzierung von Zinskosten, sondern auch die Bewertung der Unternehmen als ganze, die positiv mit ihrer Größe korreliert ist, zu nennen. Kapitalströme fließen dorthin, wo Wachstums- und Verzinsungschancen am aussichtsreichsten sind. Insbesondere kapitalmarktorientierte Unternehmen müssen, um auf den globalen Kapitalmärkten existieren zu können, permanent und kurzfristig Erfolg, Wachstum und Renditeaussichten vorweisen können.

Last but not least sind staatliche Aktivitäten ein wichtiger Einflussfaktor für die mit den wirtschaftlichen Aktivitäten verbundenen Mengenströme. Dies ist unmittelbar evident in den diversen Bereichen, in denen die Wirtschaftspolitik zur Förderung des Wirtschaftswachstums beiträgt. Dies kann aus Gründen der nationalen Wettbewerbspolitik oder der Vermeidung von beziehungsweise des Umgangs mit Konflikten zwischen Interessengruppen resultieren. $\mathrm{Zu}$ verweisen ist in diesem $\mathrm{Zu}$ sammenhang auch auf das Institut der Staatsverschuldung, durch die eine öffentlich garantierte zusätzliche Nachfragequelle entsteht.

Im Rahmen der gegenwärtig gegebenen Produktions- und Konsumweise werden diese verschiedenen Treiber für die Ausdehnung der Mengenströme zumeist über positive Rückkopplungen miteinander verknüpft und darüber eine systemische Wachstumsdynamik begründet:

- Unternehmen schaffen durch ihre Aktivitäten Einkommen und Produkte und insoweit neue Profilierungsmöglichkeiten im Konsum. Teilweise geschieht dies durch direkte Beeinflussung der Präferenzen;

- nachholende, konformistische und positionelle Bedürfnisse schaffen neue Aktivitätsmöglichkeiten für die Unternehmen und schließlich

- werden die sozialen und ökologischen Folgeprobleme der Unternehmens- und Konsumaktivitäten zum Gegenstand einer staatlichen Bearbeitung und geben insoweit Anlass für die weitere Ausdehnung staatlicher Aktivitäten.

Es scheint in der ökonomischen Nachhaltigkeitsforschung unstrittig zu sein, dass das gegenwärtig zu verzeichnende Wachstum der umweltbelastenden Mengenströme nicht zukunftsfähig ist. In welchem Ausmaß deren Reduktion zur dauerhaften Sicherstellung der Leistungsfähigkeit der Ökosysteme erforderlich ist und vor allem wie diese Reduktion adressatenspezifisch zu bewerkstelligen ist, stellt angesichts der skizzierten endogenen Wachstumsdynamik der gegenwärtigen Produktions- und Lebensweise eine zentrale Forschungsaufgabe für die Nachhaltigkeitsökonomik dar. Auch stellt sich die Frage, inwieweit bei Aufrechterhaltung des monetär gemessenen Wachstums, wenn man dieses wie oben dargelegt als systemisch angelegt annimmt, dennoch eine Reduktion der belastenden Mengenströme erreicht werden kann, indem Wachstum und Umsatz statt über materielle Produkte zunehmend über weniger materialintensive Dienstleistungen erzielt werden.

\section{Anmerkung}

Wir danken Niko Paech für Kommentare und Anregungen.

\section{AUTOREN + KONTAKT}

Dr. Frank Beckenbach ist Professor am Fachgebiet Umweltund Verhaltensökonomik der Universität Kassel.

Frank Beckenbach, Fachbereich Wirtschaftswissenschaften, Universität Kassel. Tel.: +49 561 804-3884,

E-Mail: beckenbach@wirtschaft.uni-kassel.de

Dr. Heinz Welsch ist Professor für Wirtschaftstheorie an der Universität Oldenburg.

Heinz Welsch, Fachgebiet Wirtschaftstheorie, Universität Oldenburg. Tel.: +49 441 798-4112, E-Mail:welsch@uni-oldenburg.de

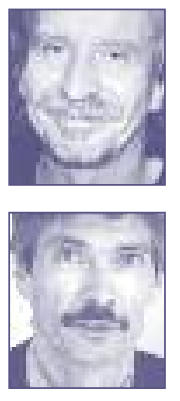


Copyright (C) 2012, IÖW und oekom Verlag. Die Nutzung des Artikels ist Abonnenten von Ökologisches Wirtschaften vorbehalten. Nachdruck und Vervielfältigung des Artikels einschließlich Speicherung und Nutzung auf optischen und elektronischen Datenträgern nur mit Zustimmung der Redaktion von Ökologisches Wirtschaften (http://www.oekologischeswirtschaften.de). 